\title{
Determination of Estrogens in Milk Using Polypyrrole Fiber-Mediated Solid-Phase Extraction Followed by High Performance Liquid Chromatography
}

\author{
Lanlan Wei, ${ }^{a, \#}$ Yan Yan, ${ }^{b, \#}$ Jianjun Deng, ${ }^{c}$ Yuqin Ma, ${ }^{c}$ Yu Wang, ${ }^{d}$ Xiaoxiao Wu ${ }^{e}$ and \\ Xuejun Kang*,d
}

${ }^{a}$ Key Laboratory of Environmental Medicine and Engineering (Ministry of Education), School of Public Health, Southeast University, 210009 Nanjing, China

${ }^{b} J i a n g s u$ Product Quality Testing \& Inspection Institute, 210000 Nanjing, China

'Suzhou Dongqi Biological Technology Co., Ltd., 215123 Suzhou, China

${ }^{d}$ Key Laboratory of Child Development and Learning Science (Ministry of Education), School of Biological Science and Medical Engineering, Southeast University, 210096 Nanjing, China

${ }^{e}$ National Center of Supervision Inspection on Processed Food \& Food Additives Quality, Nanjing Institute of Product Quality Inspection, 210019 Nanjing, China

\begin{abstract}
A solid-phase extraction (SPE) method based on conductive polypyrrole (PPy) nanofibers which were fabricated by electrospinning and in situ polymerization was developed. PPy nanofibersmediated SPE followed by high performance liquid chromatography (HPLC) was used for the determination of three synthetic estrogens, namely diethylstilbestrol (DES), dienestrol (DIS), or hexestrol (HS) in milk sample. Extraction conditions including extraction nanofibers, donor solution $\mathrm{pH}$ and salt concentration were optimized. The target compounds were extracted from a $0.5 \mathrm{~mL}$ aqueous sample at pH 5.0 through PPy fibers, and then were eluted with $0.1 \mathrm{~mL}$ methanol. After extraction, the eluant was directly injected into an HPLC system for detection. Under the optimized extraction conditions, a large enrichment factor was achieved for three estrogens. The limit of detection (LOD) at a signal to noise ratio (S/N) of 3 ranged from 0.02 to $0.05 \mu \mathrm{gL}^{-1}$ for the estrogens in milk sample.
\end{abstract}

Keywords: liquid dairy products, packed-fibers solid-phase extraction, high performance liquid chromatography, estrogens

\section{Introduction}

Estrogens have been considered as one of the groups of analytes concerned in certain food matrices, especially in milk and other dairy products. ${ }^{1}$ Synthetic estrogens such as diethylstilbestrol (DES), dienestrol (DIS) and hexestrol (HS) are potent regulatory factors in physiological response medicine, and have been used widely as growth promoters in livestock and as a treatment for estrogen-deficiency disorders in veterinary medicine.

A variety of techniques have been applied to determine estrogens. The abuse of estrogens is known to be strongly harmful to humans owing to their potential carcinogenic properties. The use of estrogens in food-producing

*e-mail: xjkang64@163.com

${ }^{*} \mathrm{~L}$. Wei and Y. Yan contributed equally to this work. animals and other products has been prohibited in many countries. Synchronously, more attention is paid to residue analysis of these estrogens that helps us control their illegal use..$^{2-4}$ Generally, the determination of DES in wastewater has been carried out using gas chromatographymass spectrometry, ${ }^{5,6}$ liquid chromatography-mass spectrometry ${ }^{7,8}$ or high performance liquid chromatography equipped with diode array detection. ${ }^{9-11}$ Since the concentration of DES in complex matrices is usually extremely low, a preconcentration step, such as solidphase extraction (SPE) and liquid-phase microextraction (LPME), is required. However, the lack of selectivity and low recovery are the main problems in the determination of estrogens by using common samples preconcentration. ${ }^{12-15}$

Solid-phase extraction, because of its advantages such as less organic solvent consumption, simplicity, high recovery rate, easy operation and automation, has been 
widely applied to the extraction of different compounds. ${ }^{16}$ Recently, a novel solid-phase extraction method based on electrospun polymer nanofibers as adsorbents to enrich target compounds has been developed. ${ }^{17}$ Owing to their large surface area to volume ratio, electrospun nanofibers facilitate the miniaturization of SPE when they are packed into a pipette tip as the sorbent beds. With this packed-fiber solid-phase extraction (PFSPE) platform, the extraction capability could be efficiently improved and the volume of desorption solvent could be reduced to microliter levels. Thus, the evaporation step is not needed any more. PFSPE has been successfully applied in pharmaceutical analysis and pharmacokinetic studies of animals and humans, analysis of functional ingredients in health foods and determination of environmental pollutants in various sample matrices, etc. ${ }^{18} \mathrm{PFSPE}$ using polystyrene (PS) nanofibers as extracting agent coupled with high performance liquid chromatography (HPLC) tandem mass spectrometry (MS/MS) has been used for the simultaneous determination of three estrogens including DES, HS and DIS in liquid dairy products, ${ }^{19}$ but the target compounds must be extracted by acetonitrile, and an evaporating step was needed to remove the organic solvent before PFSPE.

In recent years, conductive polymers have attracted a great interest for the extraction of polar compounds due to their following advantages. They have a high extraction efficiency for polar compounds because of their inherent and unusual multifunctionality, such as ion-exchange properties, the $\pi$ - $\pi$ interactions, hydrogen bonding, acid-base properties, polar functional groups, and electroactivity. ${ }^{20}$ Among the conducting polymers, polypyrrole (PPy) is especially promising for commercial applications because of its better environmental stability and facile synthesis. ${ }^{21}$ In this paper, we develop an SPE method by combining the advantages of PPy and PFSPE. DES, DIS, and HS in milk are selected as the model analytes to exhibit the potential application of the PPy fibers in the PFSPE of these molecules after a simple precipitation of protein in milk. The structures of DES, DIS and HS are given in Figure 1. Under optimized extraction conditions, the proposed method is calibrated for quantitative analysis of the target compounds in milk samples.

\section{Experimental}

\section{Reagents and chemicals}

DES, DIS and HS standards were purchased from SigmaAldrich (USA). PPy nanofibers were from Dongqi BioTechnology Co., Ltd. (China). HPLC grade methanol were from Sinopharm Chemical Reagent (China). Each estrogen was dissolved in methanol to obtain a standard solution with a concentration of $0.1 \mathrm{mg} \mathrm{mL}^{-1}$ and stored at $4^{\circ} \mathrm{C}$. The working solutions with $0.16,0.47,1.42,4.27$, and $12.80 \mu \mathrm{gL}^{-1}$ of DIS; $0.49,0.99,1.97,3.94$, and $7.89 \mu \mathrm{g} \mathrm{mL}^{-1}$ of DES; and $0.17,0.51,1.51,4.62$, and $13.85 \mu \mathrm{g} \mathrm{mL}^{-1}$ of HS were obtained by diluting the $0.1 \mathrm{mg} \mathrm{mL}^{-1}$ solution with ultrapure water.

\section{Characterization}

The morphology images of the PS nanofibers and PPy nanofibers were obtained by using a scanning electron microscope (SEM, Hitachi S-3000N, Japan), at an acceleration voltage of $10 \mathrm{kV}$ and a specific surface and porosity analyzer (Micromeritics ASAP2020, USA).

\section{Instrumentation}

Centrifuge was purchased from Ruijiang Corporation (China). XW-80A vortex mixer was from Vimins (China). PFSPE column (styrene copolymer type) and array SPE extraction device were purchased from Suzhou DongQi Biological Technology Co., Ltd. (China). The HPLC system consisted of an LC-20A pump, an SPD-M20A detector, an SIL-20AC autosampler and a CTO-20A oven purchased from Shimadzu Corporation (Japan), equipped with an analytical column (Shimadzu C18, $250 \times 4.6 \mathrm{~mm}^{2}$, $5 \mu \mathrm{m}$ ) attached to a photodiode array detector. The mobile phase consisted of $60 \%$ methanol in $20 \mathrm{mmol} \mathrm{L}^{-1}$ sodium hydrogen phosphate $(\mathrm{v} / \mathrm{v}), \mathrm{pH}$ 6.0. The flow rate was kept constant at $1.0 \mathrm{~mL} \mathrm{~min}^{-1}$ and the wavelength for $\mathrm{UV}$ detector was set at $230 \mathrm{~nm}$.

\section{Samples preparation}

Milk was purchased from a supermarket in Nanjing, China. Typical samples without detectable estrogens

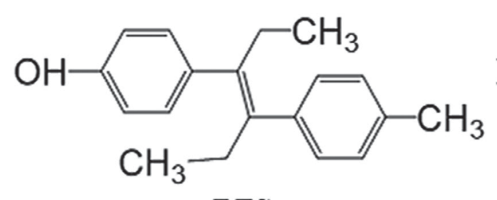

DES

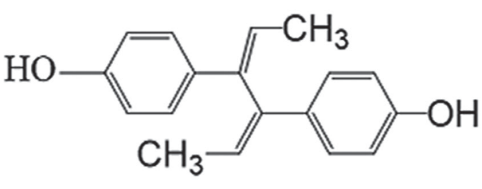

DIS

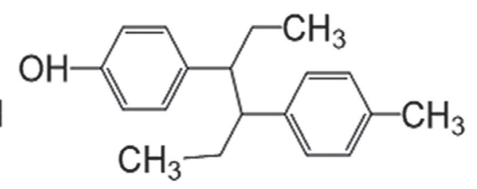

HS

Figure 1. Structural formulas of DES, DIS, and HS. 

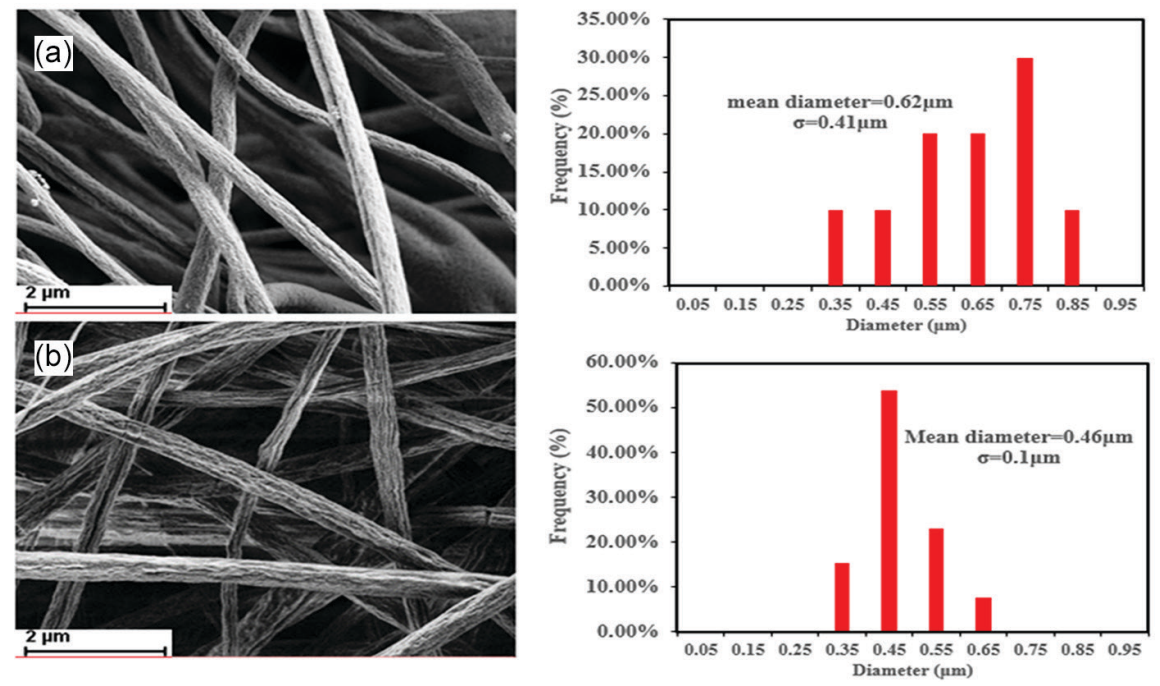

Figure 2. SEM images of (a) PPy elecrospun nanofibers and (b) PS elecrospun nanofibers.

identified by a literature method were used as the blank samples. ${ }^{22}$

The preparation of milk sample employed the following steps: (i) insertion of $1.8 \mathrm{~mL}$ milk into $4 \mathrm{~mL}$ centrifuge tube; (ii) addition of $0.2 \mathrm{~mL}$ estrogens (DIS, DES and HS) with appropriate concentration; (iii) addition of $1.0 \mathrm{~mL}$ $30 \%$ acetocaustin; (iv) sonication of the mixture for $10 \mathrm{~min}$ and centrifugation at $4000 \mathrm{rpm}$ for $10 \mathrm{~min}$, then separation of the supernatant; $(v)$ transfer of the total supernatant to clean tube and modification to $\mathrm{pH} 5$ by adding $220 \mu \mathrm{L}$ of $4.0 \mathrm{~mol} \mathrm{~L}^{-1}$ sodium hydroxide solution before the treatment of PFSPE.

The PFSPE column, as shown in literature,,$^{23}$ was preconditioned by $100 \mu \mathrm{L}$ of methanol and then $200 \mu \mathrm{L}$ of water. The total supernatant was loaded and pushed through the sorbent by the pressure of air forced by gas tight plastic syringe. Target retained on the PFSPE was eluted with $100 \mu \mathrm{L}$ of methanol and $20 \mu \mathrm{L}$ of elution was injected into the HPLC by autosampler.

\section{Results and Discussion}

\section{Morphology of PPy fibers}

The surface of PPy nanofibers synthesized under ultrasonication appears quite smooth at low magnification (Figure 2a). The nanofibers had an outer diameter of approximately $620 \pm 410 \mathrm{~nm}$. PS nanofibers with smooth and uniform morphology and random orientation were generated with a mean diameter of $460 \pm 100 \mathrm{~nm}$ (Figure 2b). As observed from the images, the presence of the nanostructure could provide a high specific surface area and many interaction sites that could offer increased SPE efficiency.
The textural properties, including the BrunauerEmmett-Teller (BET) surface area, pore volume and pore size are listed in Table 1. It can be seen that the BET surface area and pore volume of the PPy nanofibers were the lowest.

Table 1. Textural properties of the tested materials

\begin{tabular}{lcc}
\hline Material & $\begin{array}{c}\text { BET surface area / } \\
\left(\mathrm{m}^{2} \mathrm{~g}^{-1}\right)\end{array}$ & $\begin{array}{c}\text { Pore volume / } \\
\left(\mathrm{cm}^{3} \mathrm{~g}^{-1}\right)\end{array}$ \\
\hline PPy nanofibers & 10.50 & 0.02 \\
PS nanofibers & 42.31 & 0.26 \\
\hline
\end{tabular}

BET: Brunauer-Emmett-Teller; PPy: polypyrrole; PS: polystyrene.

\section{Solid-phase extraction of estrogens}

First, the effect of nanofiber characteristics on the efficiency of extraction was investigated. In this work, two types of nanofibers prepared with different framework were evaluated for their extraction recoveries. As shown in Figure 3, the extraction recoveries of PPy nanofibers for DES, DIS, HS were $27.0,35.0$, and $38.0 \%$, respectively,

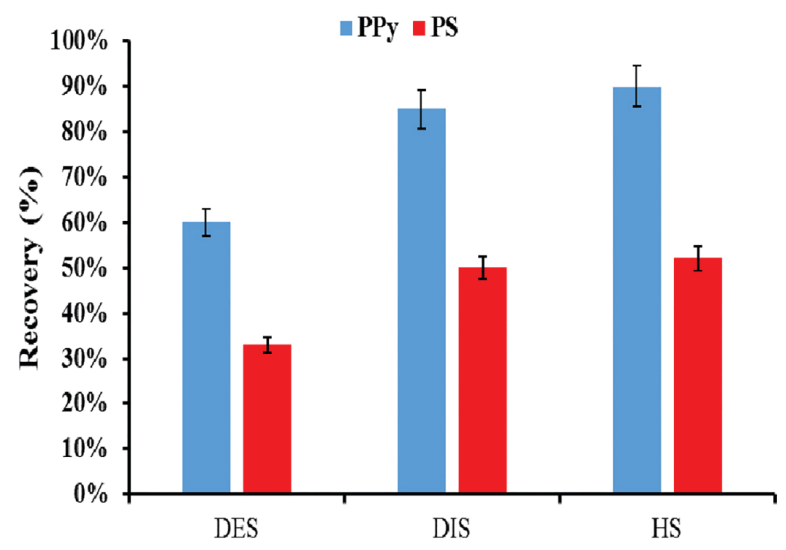

Figure 3. Influence of different materials on the extraction. 

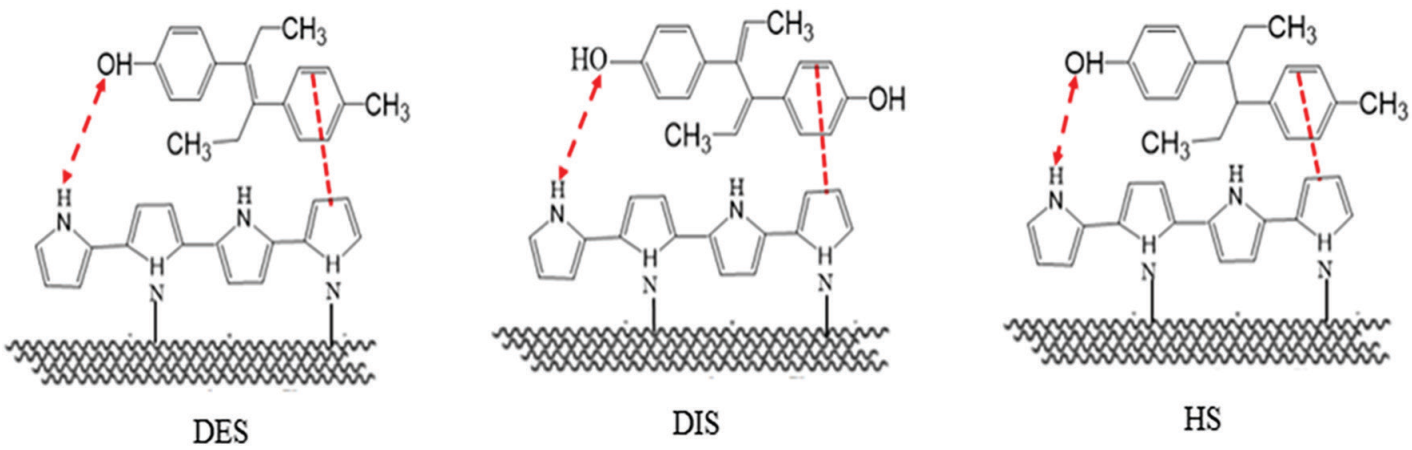

Figure 4. The interaction between estrogens and PPy nanofibers.

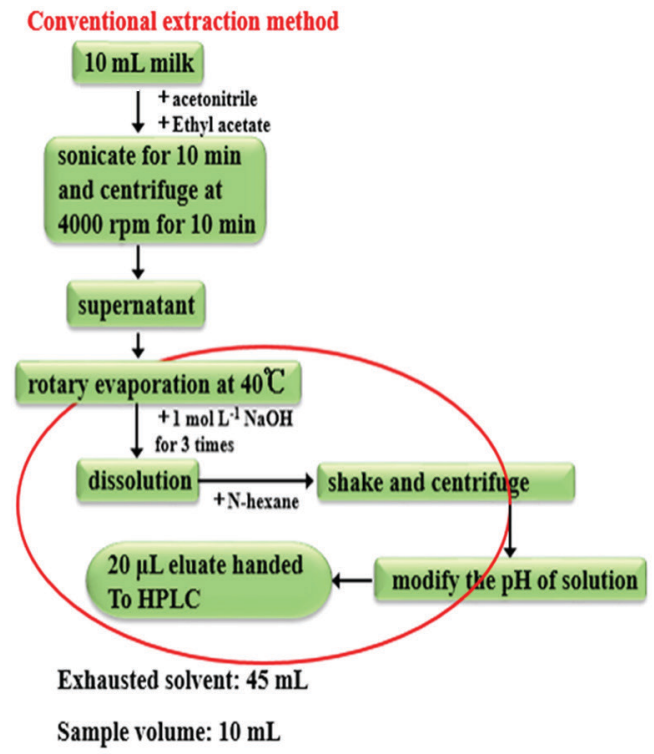

(a)

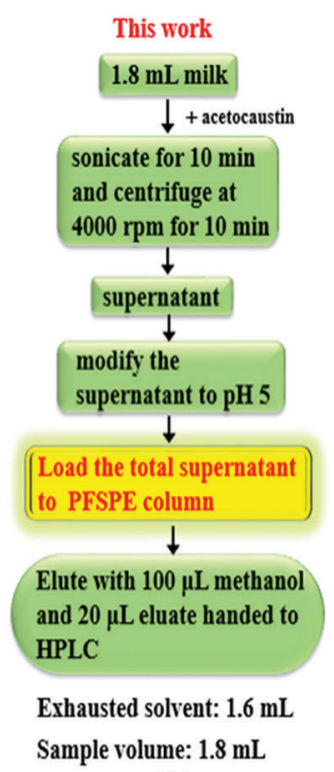

(b)

Figure 5. Flow chart of determination methods: (a) previously reported conventional extraction methods; (b) the method proposed in this paper.

which were higher than those of PS nanofibers. The PPy nanofibers diameter, however, was thicker (Figure 2) and the BET surface area was smaller (Table 1). The result could be explained by the interaction mechanism between PPy and the analytes, because the conjugated $\pi$ structure was found in PPy backbone, which showed strong interaction with analytes, and hydrogen bonding interaction could also exist between the polymer and analytes, as shown in Figure 4.

Figure 5 described the previously reported conventional extraction method for determining estrogens. Comparing with conventional methods, PFSPE method had advantages like less organic solvent consumption, simplicity, high recovery rate, easy operation and automation.

\section{$\mathrm{pH}$ of the donor solution}

To investigate the effect of $\mathrm{pH}$, the $\mathrm{pH}$ value of the donor solution was adjusted to 3.0, 4.0, 5.0 and 6.0 with $4.0 \mathrm{~mol} \mathrm{~L}^{-1} \mathrm{NaOH}$. As shown in Figure 6, an inverted $\mathrm{v}$-shaped outline of elution efficiency $v s$. the $\mathrm{pH}$ of donor solution was observed. The peak area was improved with the increasing $\mathrm{pH}$ until its highest point ( $\mathrm{pH}$ 5), after that, the peak area was decreased. Therefore, $\mathrm{pH} 5.0$ was selected.

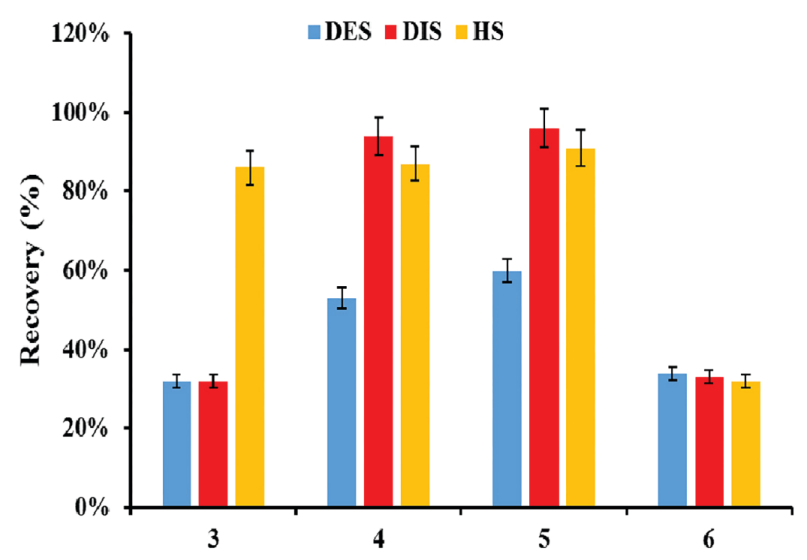

Figure 6. Effect of donor solution $\mathrm{pH}$ on the extraction. 


\section{Effect of salt concentration}

The effect of salt concentration on the donor phase was also investigated. $\mathrm{NaCl}$ is added at concentrations of $0.01,0.05,0.15$, and $0.35 \mathrm{~g} \mathrm{~mL}^{-1}$. As shown in Figure 7 , addition of $\mathrm{NaCl}$ improved the recovery of the analytes. However, the recovery in $0.15 \mathrm{~g} \mathrm{~mL}^{-1} \mathrm{NaCl}$ is lower than that in $0.05 \mathrm{~g} \mathrm{~mL}^{-1} \mathrm{NaCl}$. Two simultaneous processes could explain this phenomenon. With the addition of salt, hydration spheres are generated around the ionic salt molecules in aqueous solution. These hydration spheres reduce the amount of water which dissolves analyte molecules, resulting in additional analytes moving near the sorbent. ${ }^{24}$ At the same time, estrogen molecules may participate in electrostatic interactions with the salt in solution, thereby decreasing their ability to move into the extraction nanofibers. Initially, the predominant process would be the interaction of the salt molecules with water molecules; but as the salt concentration further increased, salt molecules would interact with analyte molecules. It seemed reasonable to add $0.05 \mathrm{~g} \mathrm{~mL}^{-1} \mathrm{NaCl}$ to the donor phase, since it contributed to the best extraction efficiency. However, in view of the salt introduced in the steps of precipitating protein and $\mathrm{pH}$ adjustment, $\mathrm{NaCl}$ was not added into the sample in the analysis of the samples.

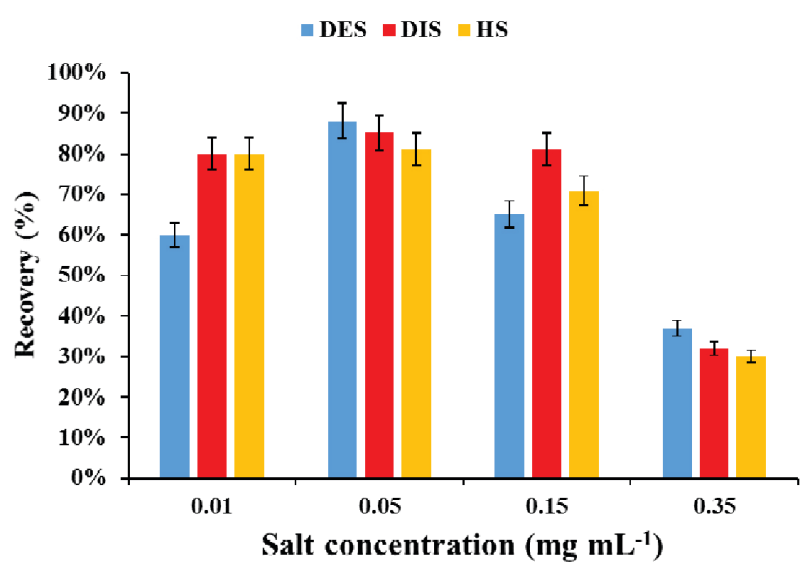

Figure 7. Effect of salt concentration on the extraction.

Validation of the method

The linearity and the limit of detection (LOD) of the method under the optimal extraction conditions were investigated (Table 2) in order to evaluate the practical applicability of the PPy approach. A good linearity was found within the range $0.49-7.89 \mu \mathrm{g} \mathrm{mL} \mathrm{m}^{-1}$ for DES, 0.17-13.85 $\mu \mathrm{g} \mathrm{mL}^{-1}$ for HS and 0.16-12.80 $\mu \mathrm{g} \mathrm{mL}^{-1}$ for DIS. Correlation coefficients $\left(\mathrm{R}^{2}\right)$ better than 0.99 were obtained. The relative standard deviations (RSD) of DES, DIS, and HS were 6.8, 9.5 and $7.9 \%$, respectively. LOD was $0.02 \mu \mathrm{g} \mathrm{mL} \mathrm{m}^{-1}$ for DIS, $0.05 \mu \mathrm{g} \mathrm{mL} \mathrm{m}^{-1}$ for DES, and $0.02 \mu \mathrm{g} \mathrm{mL}^{-1}$ for HS.

Table 2. Performance of the method

\begin{tabular}{lcccc}
\hline $\begin{array}{l}\text { Target } \\
\text { compound }\end{array}$ & $\begin{array}{c}\text { Linearity } \\
\text { range / } \\
\left(\mu \mathrm{g} \mathrm{mL}^{-1}\right)\end{array}$ & $\mathrm{R}^{2}$ & $\begin{array}{c}\text { LOD / } \\
\left(\mu \mathrm{g} \mathrm{mL}^{-1}\right)\end{array}$ & $\begin{array}{c}\mathrm{RSD} / \% \\
(\mathrm{n}=3)\end{array}$ \\
\hline DES & $0.49-7.89$ & 0.997 & 0.05 & 6.8 \\
DIS & $0.16-12.80$ & 0.997 & 0.02 & 9.5 \\
HS & $0.17-13.85$ & 0.998 & 0.02 & 7.9 \\
\hline
\end{tabular}

$\mathrm{R}^{2}$ : correlation coefficient; LOD: limit of detection; RSD: relative standard deviation; DES: diethylstilbestrol; DIS: dienestrol; HS: hexestrol.

\section{Applicability to detection in real sample}

The proposed analytical method was applied to the analysis of milk samples purchased from a supermarket in Nanjing, China, and no target analytes were detected. The samples were then spiked with DES standards at 0.8 and $1.6 \mu \mathrm{g} \mathrm{mL}^{-1}$ levels, and DIE and HS at 0.3 and $0.9 \mu \mathrm{g} \mathrm{mL}^{-1}$ levels to assess matrix effects. Estrogens were detected in milk samples (as shown in Figure 8). The recoveries from the blank milk are presented in Table 3, indicating that the influence of the matrix was not significant for food analysis.

\section{Conclusions}

A fast, sensitive HPLC-UV method for the determination of estrogens in milk was presented in this paper. Although PS nanofibers based on PFSPE coupled with HPLC-MS/MS has already been reported by our group, special and expensive equipment, even staff with a high level of operation are required. This manuscript provides a low-cost determination method with a simpler pretreatment for three estrogens in food. The PFSPE pretreatment method offered a simple, fast and low-cost operation. The microliter amount of eluting solvent could improve the conventional enrichment process, which obviously illustrated the environmental friendliness. Moreover, higher extraction recoveries and good reproducibility were especially suitable for trace analysis of the samples.

\section{Acknowledgments}

This study was supported by the National Science Foundation of China (No. 81673230, No. 21307086 and No. 81172720); the Social Development Research Program of Jiangsu Province Science and Technology department 


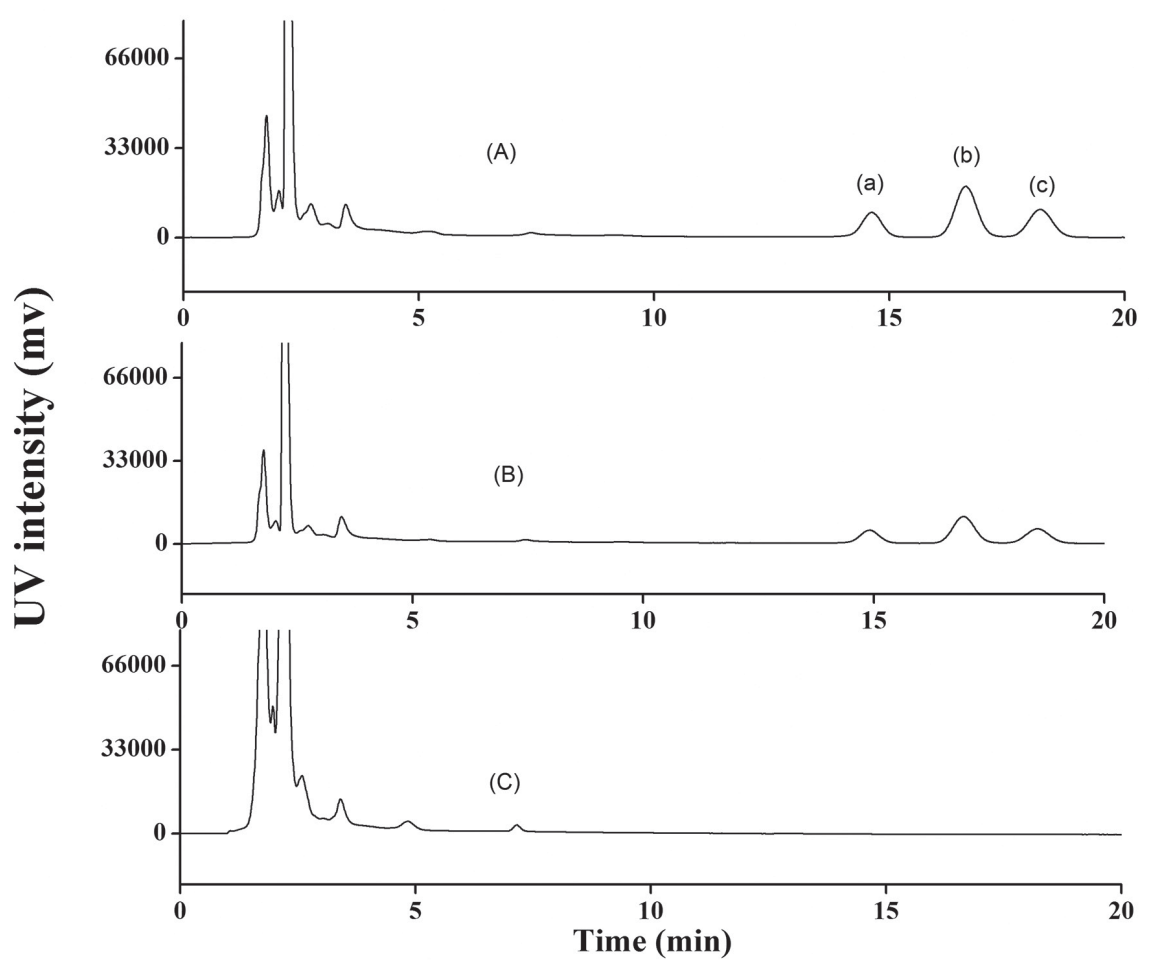

Figure 8. HPLC-UV spectra of real samples. (A) Chromatogram of standard solution after extraction; (B) chromatogram of milk sample spiked with each analyte after extraction; and (C) chromatogram of milk sample after extraction. Analytes: (a) DES, (b) DIS, and (c) HS.

Table 3. Results from the analysis of DIS, DES, and HS in milk

\begin{tabular}{|c|c|c|c|c|c|c|}
\hline Sample & DIS / $\left(\mu \mathrm{g} \mathrm{mL}^{-1}\right)$ & Recovery / \% & $\mathrm{DES} /\left(\mu \mathrm{g} \mathrm{mL}^{-1}\right)$ & Recovery / \% & $\mathrm{HS} /\left(\mu \mathrm{g} \mathrm{mL} L^{-1}\right)$ & Recovery / \% \\
\hline 1 & & - & & - & & - \\
\hline 2 & - & - & - & - & - & - \\
\hline 3 & & - & & - & & - \\
\hline 1 & & 97.5 & & 102.5 & & 100.0 \\
\hline 2 & 0.3 & 102.5 & 0.8 & 93.2 & 0.3 & 97.6 \\
\hline 3 & & 100.0 & & 104.3 & & 101.2 \\
\hline 1 & & 98.9 & & 100.0 & & 100.0 \\
\hline 2 & 0.9 & 103.4 & 1.6 & 96.9 & 0.9 & 97.8 \\
\hline 3 & & 97.7 & & 104.7 & & 101.1 \\
\hline
\end{tabular}

DES: diethylstilbestrol; DIS: dienestrol; HS: hexestrol.

(No. BE2016741); Science \& Technology Project of China General Administration of Quality Supervision, Inspection and Quarantine (No. 2015QK055); and Postgraduate Research \& Practice Innovation Program of Jiangsu Province (No. KYCX17_0189).

\section{References}

1. Farlow, D. W.; Xu, X.; Veenstra, T. D.; J. Chromatogr. B: Anal. Technol. Biomed. Life Sci. 2009, 877, 1327.

2. Pfaffl, M. W.; Reck, B.; Dreher, R.; Meyer, H. H.; Anal. Chim. Acta 2003, 483, 401.
3. Newbold, R. R.; Toxicol. Appl. Pharmacol. 2004, 199, 142.

4. Martino, M. A.; Nevadunsky, N. S.; Magliaro, T. J.; Goldberg, M. I.; Prim. Care Update Ob Gyns 2002, 9, 7.

5. Liao, S.; Wu, X.; Xie, Z.; Anal. Chim. Acta 2005, 537, 189.

6. Quintan, J. B.; Carpinteiro, I. J.; Rodríguez, R. A.; Lorenzo, A. M.; Carro-Cela, R.; J. Chromatogr. A 2004, 1024, 177.

7. Chanbasha, B.; Akhila, J.; Meng-Keow, K.; Suresh, V.; HianKee, L.; J. Chromatogr. A 2005, 1100, 137.

8. Rodriguez-Mozaz, S.; López de Alda, M. J.; Barceló, D.; J. Chromatogr. A 2004, 1045, 85.

9. Mitani, K.; Fujioka, M.; Kataoka, H.; J. Chromatogr. A 2005, 1081, 218. 
10. López de Alda, M. J.; Barceló, D.; J. Chromatogr. A 2001, 911 , 203.

11. Penalver, A.; Pocurull, E.; Borrull, F.; Marcé, R. M.; J. Chromatogr. A 2002, 964, 153.

12. Haupt, K.; Analyst 2001, 126, 747.

13. López de Alda, M. J.; Barceló, D.; J. Chromatogr. A 2001, 938, 145.

14. Thurman, E. M.; Chem. Anal. 1998, 147, 5441.

15. Kang, X.-J.; Pan, C.; Xu, Q.; Yao, Y.-F.; Wang, Y.; Qi, D.-J.; Gu, Z.-Z.; Anal. Chim. Acta 2007, 587, 75.

16. Kang, X.-J.; Chen, L.-Q.; Zhang, Y.-Y.; Liu, Y.-W.; Gu, Z.-Z.; J. Sep. Sci. 2008, 31, 3272.

17. Zhang, Y.-Y.; Kang, X.-J.; Chen, L.-Q.; Pan, C.; Yao, Y.-F.; Gu, Z.-Z.; Anal. Bioanal. Chem. 2008, 391, 2189.

18. Xu, Q.; Wang, M.; Yu, S.; Tao, Q.; Tang, M.; Analyst 2011, 136, 5030 .
19. Hu, W.-Y.; Kang, X.-J.; Zhang, C.; Yang, J.; Ling, R.; Liu, E.-H.; Li, P.; J. Chromatogr. B: Anal. Technol. Biomed. Life Sci. 2014, 957, 7.

20. Li, M.; Wei, Z.-X.; Jiang, L.; J. Mater. Chem. 2008, 18, 2276.

21. Mohammadi, A.; Ameli, A.; Alizadeh, N.; Talanta 2009, 78, 1107.

22. Boyd-Boland, A. A.; Pawliszyn, J. B.; J. Chromatogr. A 1995, 704, 163.

23. Liu, M.; Qiu, B.; Jin, X.; Zhang, L.; Chen, X.; Chen, G.-N.; J. Sep. Sci. 2008, 31, 622.

24. Zhao, R.-S.; Chu, L.-L.; Wang, Y.; Song, Y.; Liu, P.; Li, C.; Huang, J.-J.; Kang, X.-J.; Clin. Chim. Acta 2017, 468, 120.

Submitted: December 15, 2017

Published online: April 27, 2018 